Article

\title{
Glut1 and Glut3 as Potential Prognostic Markers for Oral Squamous Cell Carcinoma
}

\section{Fernanda Rocha Rojas Ayala ${ }^{1}$, Rafael Malagoli Rocha ${ }^{1, *}$, Kátia Cândido Carvalho ${ }^{1}$, André Lopes Carvalho ${ }^{2,3}$, Isabela Werneck da Cunha ${ }^{1}$, Silvia Vanessa Lourenço ${ }^{4}$ and Fernando Augusto Soares ${ }^{1}$}

1 Department of Anatomic Pathology, Hospital A.C Camargo, São Paulo, Rua Antonio Prudente, 109-1 andar, CEP: 01509-010, São Paulo, SP, Brazil

2 Department of Head and Neck, Hospital A.C Camargo, São Paulo, SP, Brazil

3 Department of Head and Neck, Hospital do Cancer, Barretos, SP, Brazil

4 Department of General Pathology, Dental School, University of São Paulo, SP, Brazil

* Author to whom correspondence should be addressed; E-Mail: rafael.malagoli@gmail.com; Tel.: 55-11-2189-1128; Fax: 55-11-2189-5103.

Received: 25 February 2010; in revised form: 24 March 2010 / Accepted: 29 March 2010 / Published: 1 April 2010

Abstract: We associated clinical-pathological features of 142 OSCC with the expression pattern of GLUT1 and GLUT3 in order to estimate their prognostic value. Methods: Clinical-pathological features and overall survival data of 142 patients with Oral Squamous Cell Carcinoma (OSCC) were retrospectively reviewed from A.C.Camargo hospital records. A tissue microarray (TMA) was built for the immunohistochemical (IHC) analysis of GLUT 1 and GLUT 3. IHC results were evaluated according to the staining pattern and number of positive cells. Results: GLUT 1 was over expressed in 50.3\% of OSSC cases showing membrane staining pattern. However, nuclear expression was observed in $49.7 \%$ of the analyzed cases. GLUT 3 over expression was detected in $21.1 \%$ of OSCC cases. The pattern of GLUT 1 expression showed significant association with alcohol consumption $(p=0.004)$. Positive cell membrane GLUT 3 protein expression was associated with advanced clinic-staging of tumours $(\mathrm{p}=0.005)$ as well as with vascular embolization $(p=0.005)$. Positive expression of GLUT 3 was associated with unfavorable free-disease survival ( $p=0.021$ ). Conclusion: GLUT1 and GLUT3 protein expression evaluated by immunohistochemistry are, significantly, indicators of poor prognosis outcome in oral 
squamous cell carcinoma, probably due to the enhanced glycolytic metabolism of more aggressive neoplastic cells.

Keywords: oral carcinoma; GLUT1; GLUT3; immunohistochemistry

\section{Introduction}

Increased glycolytic metabolism is a characteristic of malignant cells, also many mechanisms seem to contribute to the glycolytic phenotype both in normal and neoplastic cells. Since then, increased and deregulated expression of glucose transporter protein, such as GLUT1 and GLUT 3, has been associated with malignancy [1].

Some studies indicated that patients with non-small cell lung carcinoma [2], colorectal carcinoma [3], and gastric carcinoma [4], showed expression of GLUT1 and were associated with enhanced tumor aggressiveness and poor survival. Consequently, a large variety of other tumors has been related to GLUT1 and GLUT3 over expression, such as pancreatic carcinomas [5-7], cervical carcinoma [8], esophageal squamous cell carcinoma [9], and squamous cell carcinoma of the head and neck (SCCHN) [10-12].

Although GLUT1 expression is a common feature in patients with head and neck squamous cell carcinoma (HNSCC), the prognostic value of this parameter has not been analyzed systematically for this tumor type. To the best of our knowledge, only Baer and co-workers considered the prognostic significance of GLUT1 in HNSCC [5]. Additionally, the significance of GLUT1 and GLUT3 expression in oral carcinomas remains unclear and a large amount of contradictory results can be found in the literature. Based on that, our study has given further evidences of the relevant value of the glucose transporter proteins in Oral Squamous Cell Carcinoma (OSCC) evaluating GLUT1 and GLUT3 expressions using immunohistochemical techniques and correlating this expression with clinical data of patients.

\section{Results and Discussion}

\subsection{Patients’ Clinical Data}

The cohort of OSCC cases of this study included 112 male and 30 female patients (for a total of 142). The mean age of the patients was 56.6 years (range, 30-90 years) and the median was 57 years. The follow-up period of this group of patients ranged from 1 day to 157.6 months with a median of 64.9 months.

The sites of the primary tumors were, in order of frequency, as follows: tongue (54 cases or 38\%), floor of mouth (42 cases or 29.6\%), retromolar region (19 cases or 13.4\%), mandibular gingiva (14 cases or $9.9 \%$ ), maxillary gingiva (six cases or $4.2 \%$ ), buccal mucosa (six cases or $4.2 \%$ ) and palate (one case or $0.7 \%$ ). Clinical stage of tumors included: Stage I/II, 26.1\% (n = 37); Stage III/IV, 73.9\% ( $\mathrm{n}=105)$. Seventy patients $(49.3 \%)$ confirmed lymph nodes involvement. Disease outcomes recorded showed $43 \%$ of patients were alive without disease; $31.7 \%$, died through disease 
complications; $18.3 \%$ died for reasons unrelated to the disease; and $1.4 \%$, were alive with disease. Information was not available for $5.6 \%$ of cases. A summary of this data is provided in Table 1.

Table 1. Clinico-pathological information of OSCC patients.

\begin{tabular}{|c|c|c|}
\hline Varible & Group & Cases (\%) \\
\hline Gender & Male & $112(78.9)$ \\
\hline$(n=142)$ & Female & $30(21.1)$ \\
\hline Race & Caucasians & $122(85.9)$ \\
\hline$(n=142)$ & Others & $20(14.1)$ \\
\hline \multirow{4}{*}{$\begin{array}{l}\text { Tabacco } \\
(n=142)\end{array}$} & No & 15 (10.6) \\
\hline & Light & 99 (69.7) \\
\hline & Moderate/Severe & $17(12)$ \\
\hline & Ignored & $11(7.7)$ \\
\hline \multirow{4}{*}{$\begin{array}{c}\text { Alcohol } \\
(n=142)\end{array}$} & No & $27(19.0)$ \\
\hline & Light & 89 (62.7) \\
\hline & Moderate/Severe & 15 (10.6) \\
\hline & Ignored & $11(7.7)$ \\
\hline \multirow{6}{*}{$\begin{array}{c}\text { Site } \\
(n=142)\end{array}$} & Tongue & $54(38)$ \\
\hline & Gingiva & $20(14.1)$ \\
\hline & Floor of the mouth & 42 (29.6) \\
\hline & Palate & $1(0.7)$ \\
\hline & Jugal & $6(4.2)$ \\
\hline & Retromolar & 19 (13.4) \\
\hline Vascular Invision & No observed & 75 (52.8) \\
\hline \multirow[t]{2}{*}{$(n=142)$} & Yes & 53 (37.3) \\
\hline & Ignored & $14(9.9)$ \\
\hline \multirow{2}{*}{$\begin{array}{c}\text { Clinic Stage } \\
(\mathrm{n}=142) \\
\end{array}$} & $\mathrm{I} / \mathrm{II}$ & $37(26.1)$ \\
\hline & III/IV & $105(73.9)$ \\
\hline \multirow{2}{*}{$\begin{array}{c}\text { Recurrence } \\
(\mathrm{n}=142)\end{array}$} & No & $92(64.8)$ \\
\hline & Yes & $50(35.2)$ \\
\hline \multirow{4}{*}{$\begin{array}{l}\text { Site of Recurrence } \\
\qquad(\mathrm{n}=142)\end{array}$} & Local & $41(28.8)$ \\
\hline & Others & $7(4.9)$ \\
\hline & Local/others & $2(1.4)$ \\
\hline & No recurrence & 92 (64.8) \\
\hline \multirow{5}{*}{$\begin{array}{l}\text { Status of last contact } \\
\qquad(\mathrm{n}=142)\end{array}$} & Alive wth disease & $2(1.4)$ \\
\hline & Alive wthout disease & $61(43)$ \\
\hline & Death due to disease & 45 (31.7) \\
\hline & Death due to other causes & 26 (18.3) \\
\hline & Lost & $8(5.6)$ \\
\hline
\end{tabular}

\subsection{GLUT1 and GLUT3 Immunohistochemical Expression}

Immunohistochemical analyses showed GLUT1 expression in 135/142 cases, with clearly stronger and more extensive staining in neoplastic tissue than in normal adjacent hyperplastic epithelia. In regarding to GLUT1 protein pattern, a membrane pattern was seen in 68 of 135 (50.3\%), whereas a nuclear expression appeared in 67 of 135 cases (49.7\%, Figure 1). 
Figure 1. A) GLUT1 membrane immunostaining in oral squamous cell carcinoma, 400×; B) GLUT1 expression in oral squamous cell carcinoma. Note both GLUT1 expression: membrane and nucleus (arrows), 200×; C) GLUT3 membrane immunostaining in oral squamous cell carcinoma; D) No GLUT3 immunostaining. Note also GLUT3 expression in the inflammatory cells, $200 \times$.
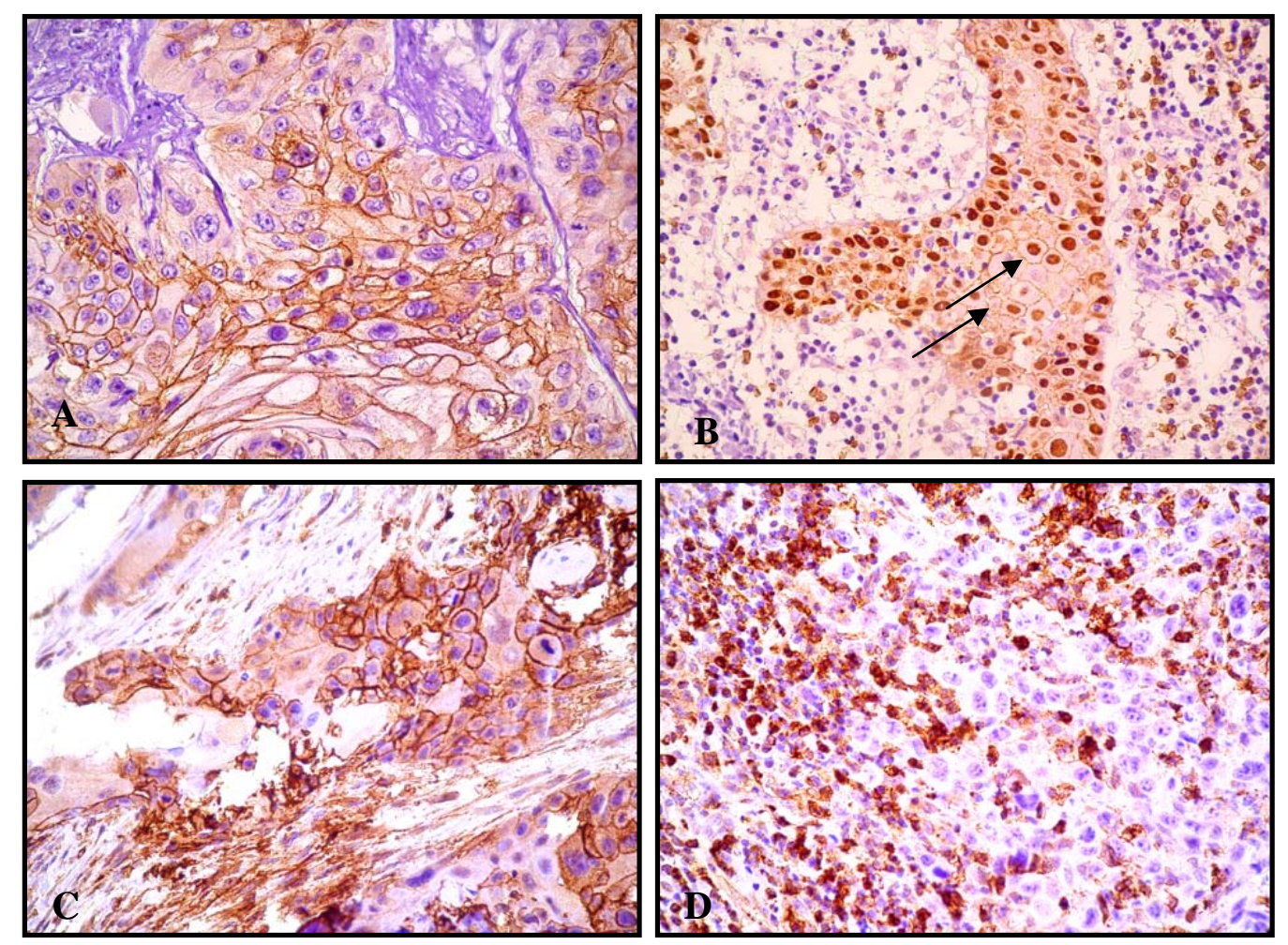

According to the number of positive cells, GLUT1 partial expression was observed in 58 cases (40.8\%), while its total frequency expression was seen in 77 cases (54.2\%). Interestingly, GLUT1 expression was observed in the majority of poorly differentiated cases, which showed an "antistromal" pattern - enhanced staining in central and perinecrotic zones. Conversely, well-differentiated tumors showed an absent GLUT1 staining in the central zones of the tumor, with its expression localized in the peripheral neoplastic area.

On the other hand, in non-neoplastic squamous epithelium, GLUT1 staining was usually undetectable or was weakly detected in supra-basal layers. Thus, a range of staining patterns was observed within the epithelia; in this manner, many cases showed a predominantly basal staining with a superficial layer showing either little or no GLUT1 staining (Figure 2).

Alternatively, GLUT3 expression that was evaluated in all 142 cases showed expression in 30 cases (21.1\%), while 112 cases (78.9\%) was negative. Notably, a strong GLUT3 immunoexpression was observed in all inflammatory cells adjacent to neoplastic islands, which were considered as an internal control (Figure 1). 
Figure 2. A) Keratinizing squamous carcinoma. GLUT1, prostromal staining pattern. Note positivity at periphery of tumour nest, in non-keratinizing basaloid cells, and loss of GLUT1 expression accompanying keratinization at the centre of tumour nest, 200×; B) $400 \times$; C) Non - keratinizing poorly differentiated carcinoma, 100×. In the absence of squamous differentiation/keratinization: GLUT1 displays an antistromal pattern, suggestive of hipoxia-driven GLUT1 induction. D) and E) Basal staning pattern with the superficial layers showing little or no GLUT1 staining in the normal epithelium; F) GLUT1 immunostain (arrow). Squamous intraepithelial neoplasia, non- staining of basal layer.

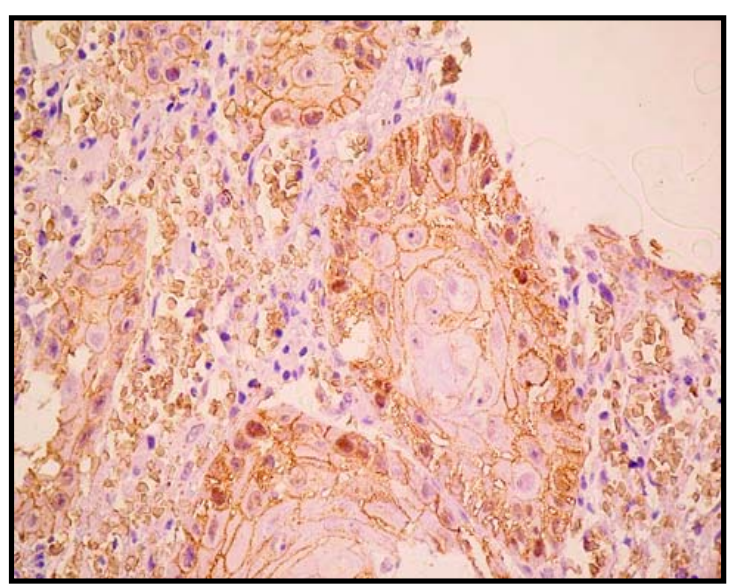

A
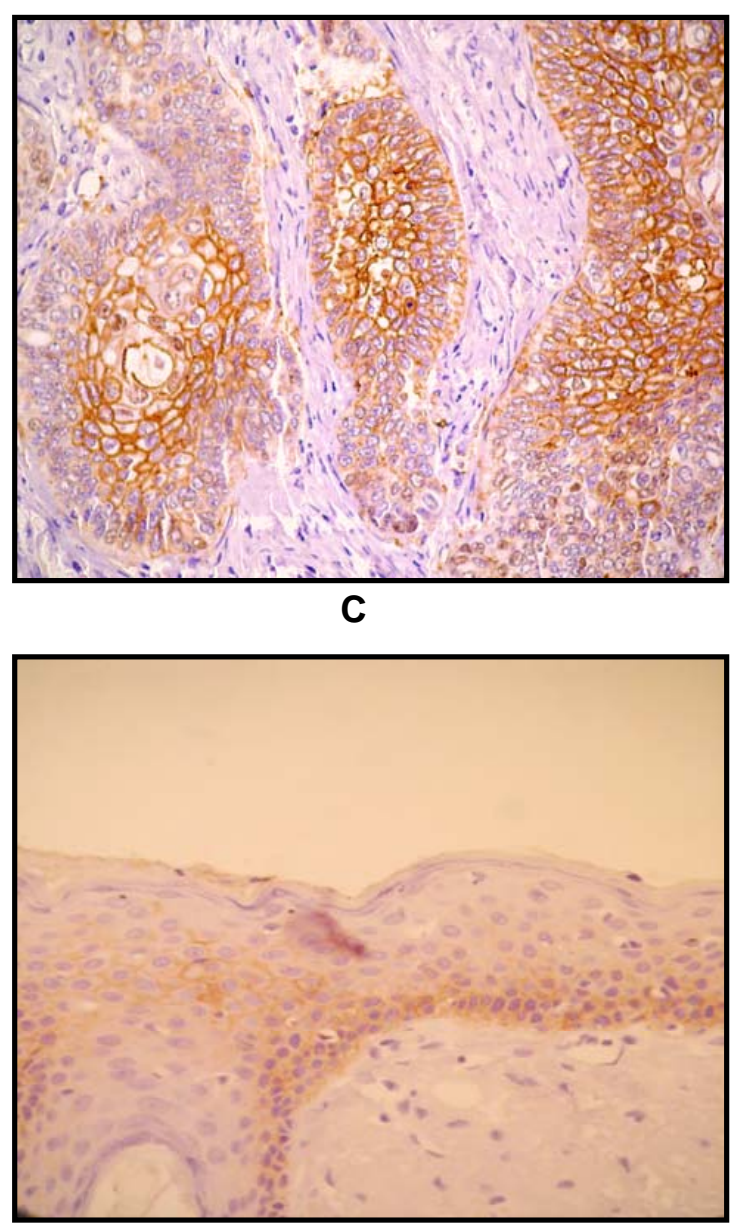

E

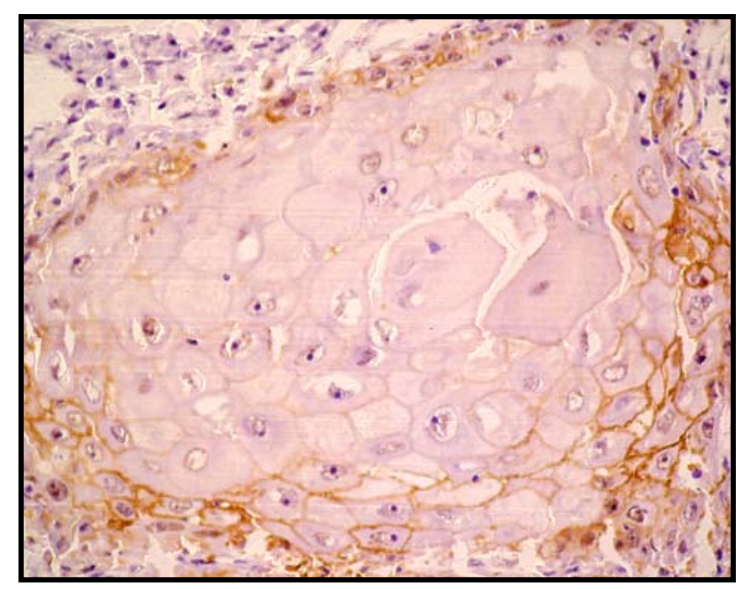

B
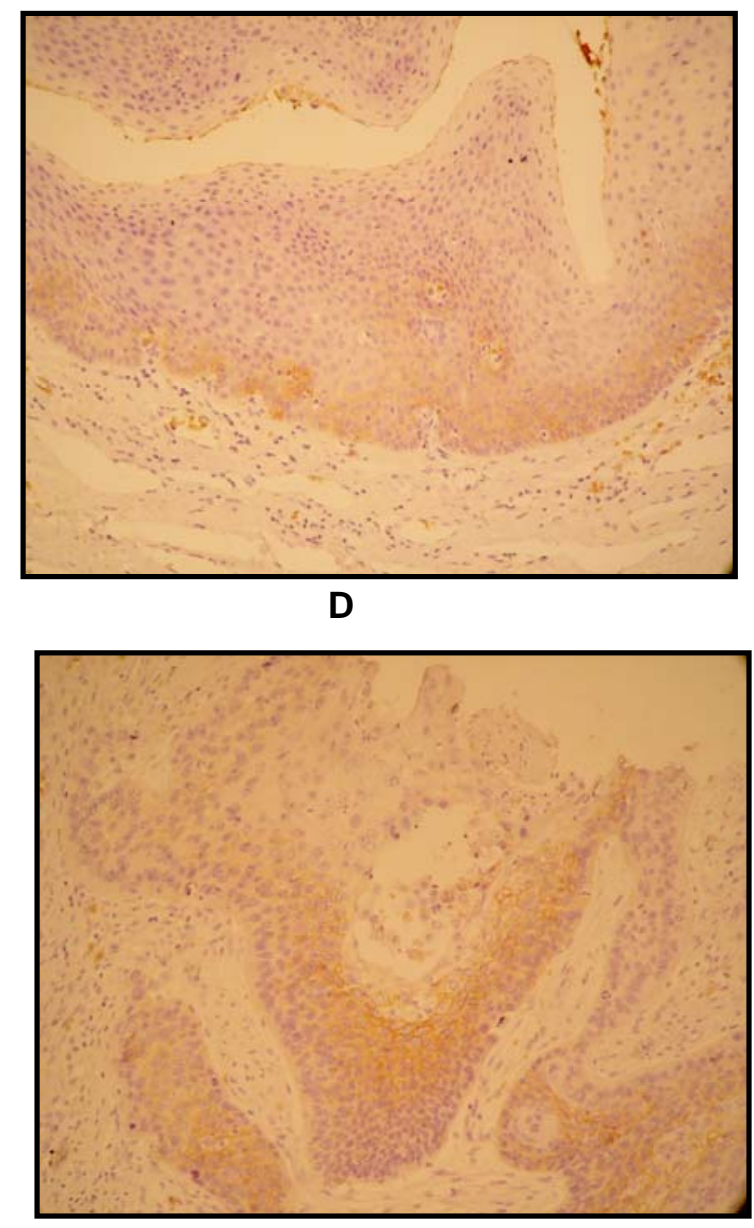

F 


\subsection{GLUT 1 and GLUT 3: Immunoexpression and Clinical-Pathological Parameters}

Univariate statistical analysis showed a significant association of cases with a reported history of alcohol consumption with GLUT1 staining nuclear pattern $(p=0.042)$. In relation to the GLUT1 frequency of cells stained, statistically significant results were found with: gender $(p=0.001)$, alcohol consumption $(p=0.030)$ and clinic tumor stage $(p=0.028)$ (Table 2$)$. In respect to GLUT3 analysis, its expression showed a significant relationship with clinic tumor stage $(p=0,005)$ and to vascular embolization $(p=0.005)$ (Table 3$)$.

Table 2. Association between GLUT1 expression and clinic-pathological feature in OSCC.

\begin{tabular}{ccccc}
\hline Variable & & \multicolumn{3}{c}{ GLUT-1/grouping } \\
$(\mathbf{n}=\mathbf{1 3 5})$ & Category & $\mathbf{M}$ & $\mathbf{M N} / \mathbf{N}$ & $\mathbf{p}$ \\
& & & $\mathbf{O R}$ & \\
& & & $\mathbf{N}$ & \\
\hline Gender & Male & $53(39.3)$ & $54(40)$ \\
& Female & $15(11.1)$ & $13(9.6)$ & 0.832 \\
\hline Alcohol & No & $14(11.3)$ & $11(8.9)$ \\
& Light & $37(29.8)$ & $48(38.7)$ & 0.042 \\
& Moderate/severe & $11(8.9)$ & $3(2.4)$ & \\
\hline Staging & I/II & $19(14.1)$ & $16(11.9)$ \\
& III/IV & $49(36.3)$ & $51(37.8)$ & 0.695 \\
\hline Vascular Embolization & No & $31(25.6)$ & $38(31.4)$ \\
& yes & $29(24)$ & $23(19)$ & 0.273 \\
\hline
\end{tabular}

M- Membranaee; MN/N: Membranaee nucleus /nucleus; p: value.

Table 3. Association between GLUT3 expression and clinic-pathological feature in OSCC.

\begin{tabular}{ccccc}
\hline \multirow{2}{*}{ Variable (n = 142) } & Category & Positivo(\%) & Negativo(\%) & p \\
\hline \multirow{2}{*}{ Gender } & Male & $87(61.3)$ & $25(17.6)$ & 0.619 \\
& Female & $25(17.6)$ & $5(3.5)$ & \\
\hline Alcohol & No & $3(2.3)$ & $24(18.3)$ & 0.316 \\
& Light & $22(16.8)$ & $67(51.1)$ & \\
& Moderate/severe & $3(2.3)$ & $12(9.2)$ & 0.279 \\
\hline \multirow{2}{*}{ Tabacco } & No & $1(0.8)$ & $14(10.7)$ & \\
& Light & $24(18.3)$ & $75(57.3)$ & 0.005 \\
\hline Staging & Moderate/severe & $3(2.3)$ & $14(10.7)$ & \\
& I/II & $2(1,4)$ & $35(24.6)$ & 0.005 \\
\hline Vascular & III/IV & $28(19,7)$ & $77(54.2)$ & \\
\hline Embolization & No & $10(7,8)$ & $65(50.8)$ & $34(26.6)$ \\
\hline
\end{tabular}

p: p value. 
It was noteworthy that for both proteins no significant association was found with primary site and lymph node involvement. Yet, no significant statistical relationship was found between GLUT 1 and GLUT 3 expression at OSCC tumors.

Concerning to these patients' outcome, poor survival rate was significantly associated with the GLUT1 nuclear expression ( $\mathrm{p}=0.015$ ) (Figure 3). Likewise, GLUT3 expression showed a strong impact on overall survival $(\mathrm{p}=0.002)$, poorer survival curves were verified in patients that showed GLUT3 expression (Figure 3). At the same way, in disease-free survival curves, we observed a statistical difference with GLUT3 expression ( $p=0.021$ ); clearly, patients that showed presence of GLUT3 revealed higher risk of recurrence (Figures 4 and 5).

Figure 3. a) Kaplan -Meier curve of overall survival at five years in patients with OSCC (52.1\%); b) Overall survival as a function of GLUT1 staining pattern ( $p=0.015)$; c) Overall survival as a function of GLUT1 frequency, according to stratified cells ( $p=0.041)$; d) Overall survival as a function of GLUT3 staining pattern (negative or positive) $(p=0.002)$.

a)

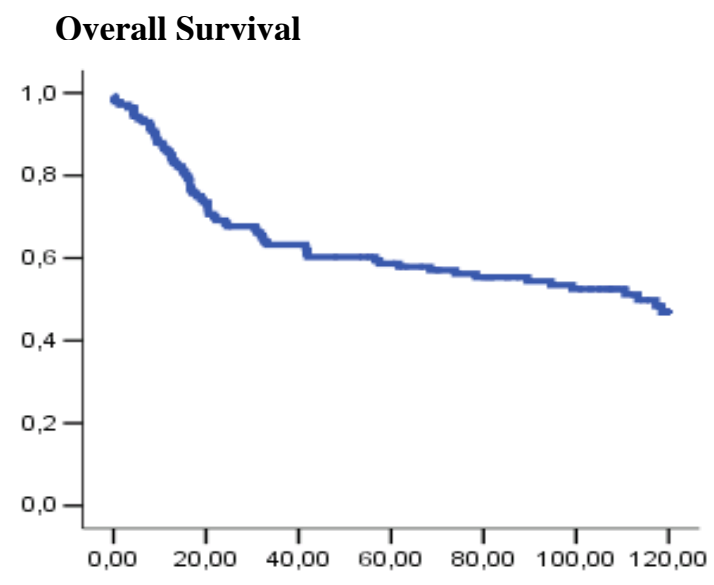

Time (months)

b)

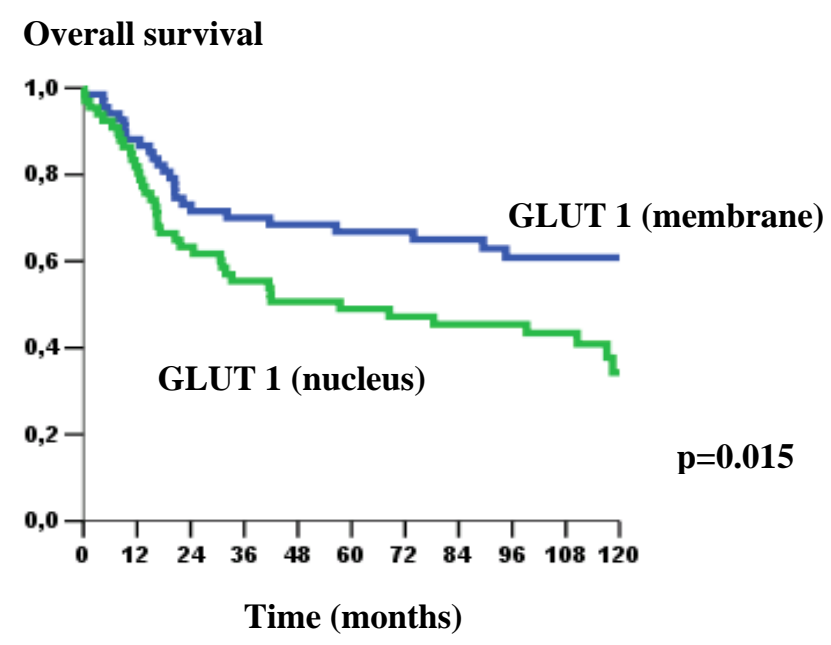


Figure 3. Cont.

Overall survival

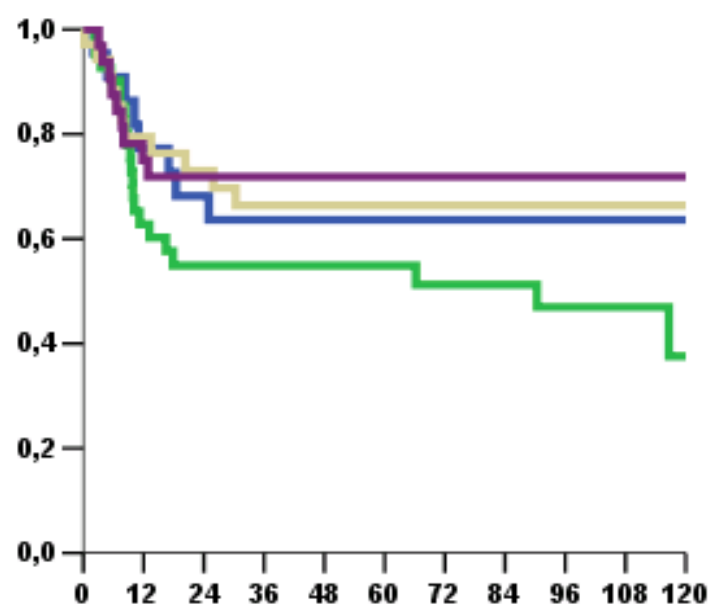

Time (months)

c)

\section{GLUT-1:}

A: Membrane total

B: Membrane partial

C: Nucleus partial

D: Nucleus total

d)

\section{Overall survival}

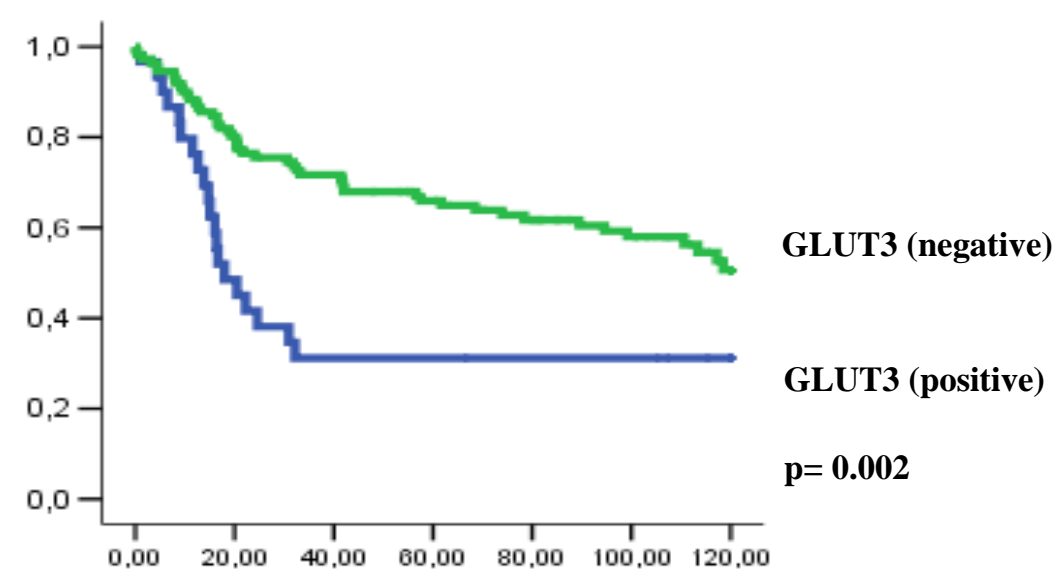

Time (months)

Figure 4. Kaplan-Meier curve of recurrence-free survival in 5 year in OSCC (64.8\%).

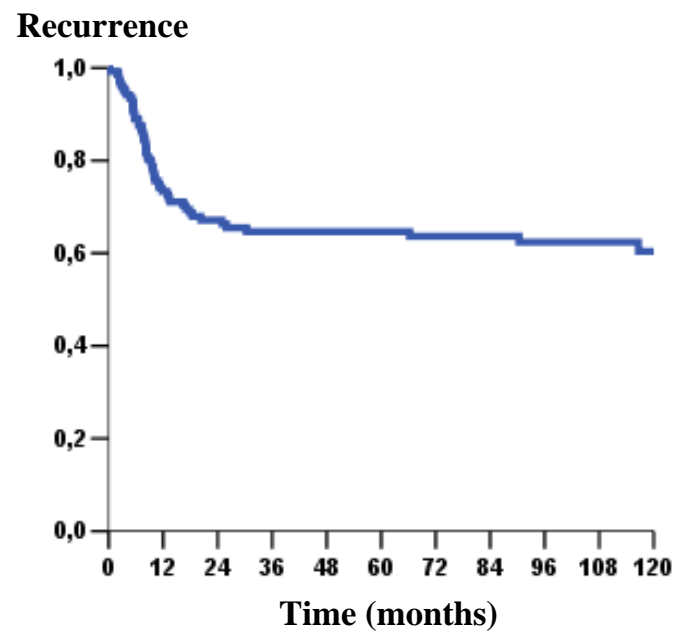


Figure 5. Kaplan - Meier curve of disease - free survival as a function of GLUT3 staining pattern $(p=0.021)$.

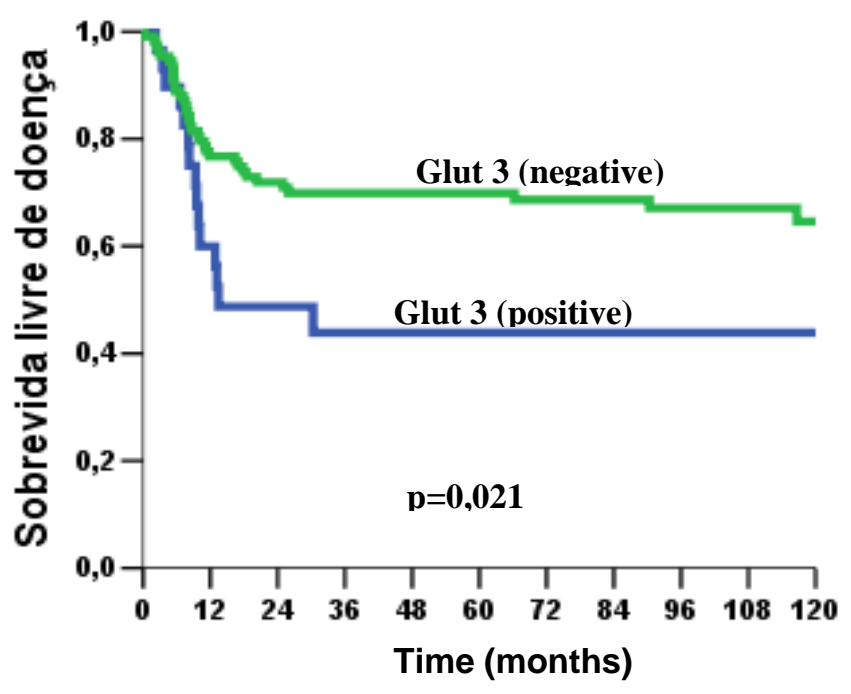

To a great extent, multivariate analysis confirmed GLUT1 expression, GLUT3 expression and vascular embolization as significant markers of independent prognostics for overall survival. Also, the clinic tumor stage was maintained as an independent prognostic marker for disease-free survival. The following hazard ratios were assigned: high GLUT1 expression, 2.066, ( $p=0.006)$; GLUT3 expression, 1.933; $\mathrm{p}=0.018)$; vascular embolization, 2.165, $\mathrm{p}=0.002$ and clinic stage, 3.304, $(\mathrm{p}=0.006)$ (Tables 4 and 5).

Table 4. Multivariate analysis by Cox regression model: Overall survival.

\begin{tabular}{llll}
\hline Variable & Category & HR (95\% IC) & p valor \\
\hline Vascular embolization & No & 1.0 (ref.) & \\
\hline GLUT-1 & Yes & $2.165(1.295-3.618)$ & 0.003 \\
\hline GLUT-1/pattern & (group) & 1.0 (ref.) & \\
\hline GLUT-3 & (group 1) & $2.066(1.232-3.464)$ & 0.006 \\
\hline & Negative & 1.0 (ref.) & \\
\hline
\end{tabular}

GLUT-1/ pattern; M- Membranaee; MN/N: Membranaee - nucleus/ nucleus.

Table 5. Multivariate analysis by Cox regression model: Overall survival: Disease-free survival.

\begin{tabular}{llll}
\hline Variable & Category & HR (95\% IC) & p valor \\
\hline staging & & & \\
& I/II & 1.0 (ref.) & \\
& III/IV & $3.304(1.407-7.762)$ & $\mathbf{0 . 0 0 6}$ \\
\hline
\end{tabular}

The enhanced expression of GLUTs proteins and the increased glucose metabolism have been previously investigated in a variety of tumors; however, poorly is known about these proteins in OSCC. Our cohort of 142 cases showed significant associations of GLUT1 and GLUT3 with clinicalpathological features of OSCC patients, which led to the light of their importance as prognostic value in this tumor type. In addition, GLUT1 protein expression confirmed to be a common characteristic of OSCC, whilst GLUT 3 expression was detected only in a limited number of specimens. 
The glucose transporters proteins, GLUT 1 and GLUT 3, largely mediate basal glucose transport in cancer cells, facilitating the maintenance of glycolytic energy metabolism in cases of limited supply of the substrate in moderately to poorly perfused regions [1]. Also, the expression of these proteins might reflect the activity of other oncogenic pathways independent of hypoxia [13].

From the aforementioned results, the GLUT1 staining was more evident in the central neoplastic nests as well as around necrotic areas. This distant localization from the supply of blood vessels naturally reinforces the concept of the existence of hypoxia-induced GLUT1 [14-16].

Also, our findings concur with the Reisser et al. [17] study, which demonstrated the presence of GLUT1 in the outer layers of tumor nests, attributing this staining pattern to differentiation functions of this protein, which in intraepithelial lesions was predictive of the differentiation status associated with invasive carcinoma [7].

In regarding to protein staining pattern, GLUT1 nuclear expression was associated with parameters of poor prognosis, supported by other study [4], this might be an additional clue to understanding the importance of this protein, not only in glucose transport, but also in other cascades of the cell machinery.

In contrast to GLUT1 expression, the GLUT3 protein was positive in only 30/142 cases (21.1\%). However, we observed a strong GLUT3 expression in all inflammatory cells adjacent to the tumor. Mochizuki et al. [8], also found GLUT1 and GLUT3 highly expressed in inflammatory tissues, besides malignant tumors. Yet, this study showed that while GLUT1 expression was significantly more frequent in tumor tissue than in inflammatory tissue, while GLUT3 expression tended to be higher in inflammatory lesions. This finding may reflect the involvement of GLUT3 in the earlier stages of carcinogenesis of some tumors.

As referred to clinical parameters, our results showed that GLUT1 pattern expressions was associated to alcohol consumption, advanced tumor staging and nodal metastasis, which corroborated with the clinical parameters observed in other malignancies such as esophageal carcinomas $[17,18]$, suggesting a connection of GLUT1 with poor prognosis.

Hence, from our records, in conformity with other studies, lead us to believe that GLUT3 expression may be related to clinic stage of OSCC patients $(p=0.005)[5,16]$.

Furthermore, our multivariate analysis findings confirmed a relationship of both proteins to patients that presented low survival rates, signifying that GLUT1 and GLUT3 may indicate a background of poor prognosis. This relationship showed consistency with studies in laryngeal carcinomas that emphasized the importance of GLUT3 as a predictor of poor prognosis, but no value for GLUT1 as a prognostic indicator [5]. However, GLUT1, which is invariably expressed in head and neck tumors, demonstrated the relationship between intense GLUT1 expression in OSCC with the presence of regional metastasis and disease-related death [14].

\section{Experimental}

\subsection{Patients}

One hundred and forty two patients diagnosed with OSCC were selected for the study. All these patients underwent surgical resection in the Head and Neck Department of A.C. Camargo Hospital of São Paulo, Brazil. Clinical information was retrospectively gleaned from patients’ records including 
demographic data, surgical resection and clinical outcome. Each case was reanalyzed and diagnoses were confirmed. H\&E slides were evaluated in order to select representative tumor areas for the TMA construction.

\subsection{Tissue microarray construction (TMA)}

Areas to be used for the TMA construction were selected on the HE slide and on the correspondent donor block of each case. The tissues corresponding to selected areas were sampled using a manual arraying instrument (Manual Tissue Arrayer 1, Beecher Instruments, Sun Prairie, WI, USA). The sampling consisted of two cores from different areas of the tumor, placed coordinately in the array. Separate samples of normal oral mucosa were used as controls. After the arraying was completed, TMA blocks were sectioned at the thickness of $4 \mu \mathrm{m}$. One section was stained with hematoxylin and eosin to check for the presence of neoplastic structures, and two other slides, distant $40 \mu \mathrm{m}$ from each other were used for the immunohistochemical study.

\subsection{Immunohistochemistry}

Four $\mu \mathrm{m}$ sections from the TMA containing 142 OSCC specimens were re-hydrated and incubated in citrate buffer, $\mathrm{pH} 6.0$ in a pressure pan for antigen retrieval. Incubation in 3\% aqueous hydrogen peroxide for $30 \mathrm{~min}$ followed to quench endogenous peroxidase activity. Incubation with $1 \%$ bovine serum albumin (BSA) and 5\% fetal calf serum (FCS) in Tris-HCl pH 7.4 for 60 min at room temperature was performed to suppress non-specific binding of subsequent reagents. The sections were then incubated with the polyclonal rabbit anti-human GLUT1 antibody (DAKO, Carpenteria, CA, USA) used at 1/500, and with polyclonal rabbit anti-human GLUT3 antibody (Lab Vision Corporation, Fremont, CA, USA) at $1 / 400$, for $2 \mathrm{~h}$ at room temperature. The reaction followed with incubation of the sections with the Advance Kit (DAKO) (ROCHA et al., 2009). Staining was completed by incubation with 3,3-diaminobenzidine tetrachloride (DAB) for $3 \mathrm{~min}$. The sections were then lightly counterstained with Mayer's hematoxylin, dehydrated and mounted with glass coverslip and xylene based medium.

Negative controls were obtained by incubating the sections with non-immune serum. Erythrocytes, which were present in every section, served as internal controls for GLUT1 and inflammatory cells of adjacent tumor were used as internal controls for GLUT3. All immunohistochemical reactions were carried out in duplicates. Two pathologists analyzed the results simultaneously using a conventional optical multihead microscope equipped with a digital camera for photographic registration. Disagreements were assessed by consulting a third expert pathologist (IWC).

\subsection{Evaluation of stained sections: GLUT1 and GLUT3}

Cellular patterns of staining and the number of positive cells were recorded for every core included in the OSCC TMA. For GLUT1, we considered positive those cases in which at least $10 \%$ of the membrane and nucleus were stained moderate. The positivity was further evaluated according to the area stained: totally positive (more than $85 \%$ of neoplastic cells stained) or partial positive (less than $85 \%$ of neoplastic cells stained). For GLUT3, only the presence or absence of moderate protein expression on the membrane of at least $10 \%$ of tumor cells was considered positive. For statistical 
analysis, the results obtained in the microscopic analysis were grouped in two categories: $\mathrm{M}$ membrane and $\mathrm{N}$ - nucleus.

\subsection{Statistical analysis}

All statistical analysis were performed using the SPSS 13.0 statistical software program (SPSS, Chicago, IL). The chi - square test and Fisher's exact test were used to analyze the association between clinical-pathological parameters and molecular biomarker immunoexpression. The five-year survival rates were estimated using the Kaplan-Meier's method, and the log-rank test was used to compare the curves. Cox proportional hazards model was performed to find independently risk factors for death. For all tests, alpha error was set at $5 \%$.

\section{Conclusions}

Taken all together, GLUT1 and GLUT3 protein expression evaluated by immunohistochemistry appear to define a small clinic subset of OSCC patients with distinctly poor outcome. The enhanced glycolytic metabolism of these proteins at more aggressive tumor cells indicates potential prognostic value in stratifying patients for risk.

\section{Acknowledgements}

Financial support was provided by Coordenação de Aperfeiçoamento de Pessoal de Nível Superior (CAPES), Fundação de Apoio à Pesquisa do Estado de São Paulo (FAPESP), and Conselho Nacional de Desenvolvimento Científico e Tecnológico (CNPq).

\section{References}

1. Kallinowski, F.; Schlenger, K.H.; Runkel, S.; Kloes, M.; Stohrer, M.; Okunieff P.; Vaupel, P. Blood flow, metabolism, cellular microenvironment and growth rate of human tumor xenography. Cancer Res. 1989, 49, 3759-3764.

2. Reisser, C.; Eichhorn, K.; Herold-Mende, C.; Born, A.I.; Bannasch, P. Expression of facilitative glucose transport proteins during development of squamous cell carcinomas of the head and neck. Int. J. Cancer 1999, 80, 194-198.

3. Haber, R.S.; Rathan, A.; Weiser, K.R.; Pritsker, A.; Itzkowitz, S.H.; Bodian, C.; Slater, G.; Weiss, A.; Burstein, D.E. GLUT 1 Glucose transporter Expression in colorectal carcinoma: a marker for poor prognosis. Cancer 1998, 17, 34-40.

4. Kunkel, M.; Reichert, T.E.; Benz, P.; Lehr, H.A.; Jeong, J.H.; Wieand, S.; Bartenstein, P.; Wagner, W.; Whiteside, T.L. Over expression of GLUT 1 and increased glucose metabolism in tumors are associated with a poor prognosis in patients with oral squamous cell carcinoma. Cancer 2003, 15, 1015-1024.

5. Baer, S.; Casaubon, L.; Schwartz, M.R.; Macrogliese, A.; Younes, M. GLUT 3 expression in biopsy specimens of laryngeal carcinoma is associated with poor survival. Laryngoscope 2002, 112, 393-396. 
6. Airley, R.; Loncaster, J.; Davidson, S.; Bromley, M.; Roberts, S.; Patterson, A.; Hunter, R.; Stratford, I.; West, C. Glucose transporter GLUT 1 expression correlates with tumor hypoxia and predicts metastasis-free survival in advanced carcinoma of the cervix. Clin. Cancer Res. 2001, 7, 928-934.

7. Burstein, D.E.; Nagi, C.; Kohtz, D.S.; Lumerman, H.; Wang, B.Y. Immunohistochemical detection of GLUT1, p63 and phosphorylated histone $\mathrm{H} 1$ in head and neck squamous intraepithelial neoplasia: evidence for aberrations in hypoxia-related, cell cycle-and stem - cellregulatory pathways. Histopathology 2006, 48, 708-716.

8. Mochizuki, T.; Tsukamoto, E.; Kuge, Y.; Kanegae, K.; Zhao, S.; Hikosaka, K.; Hosokawa, M.; Kohanawa, M.; Tamaki, N. FDG uptake and glucose transporter subtype expressions in experimental tumor and inflammation models. J. Nucl. Med. 2001, 42, 1551-1555.

9. Kato, H.; Takita, J.; Miyazaki, T.; Nakajima, M.; Fukai, Y.; Masuda, N.; Fukuchi, M.; Manda, R.; Ojima, H.; Tsukada, K.; Kuwano, H. GLUT 1 glucose transport expression in esophageal squamous cell carcinoma is associated with tumor aggressiveness. Anticancer Res. 2002, 22, 2635-2639.

10. Mellanen, P.; Minn, H.; Grenman, R.; Harkonen, P. Expression of glucose transporters in headand-neck tumors. Int. J. Cancer 1994, 56, 622-629.

11. Higashi, T.; Tamaki, N.; Honda, T.; Torizuka, T.; Kimura, T.; Inokuma, T.; Ohshio, G.; Hosotani, R.; Imamura, M.; Konishi, J. Expression of glucose transporters in human pancreatic tumors compared with increased FDG accumulation in PET study. J. Nucl. Med. 1997, 38, 1337-1344.

12. Marom, E.M.; Aloia, T.A.; Moore, M.B.; Hara, M.; Herndon, J.E.; Harpole, D.H.; Goodman, P.C.; Patz, E.F. Correlation of FDG PET imaging with GLUT 1 and GLUT 3 expression in earlystage non-small cell lung cancer. Lung Cancer 2001, 33, 99-107.

13. Gjedde, A. Glucose metabolism. In Principles and Practice of Nuclear Medicine, 2th ed.; Wagner. H., Ed.; W B Saunders: Philadelphia, PA, USA, 1995; pp.56-62.

14. Oliver, R.J.; Woodwards, R.T.; Sloan, P.; Thakker, N.S.; Stratford, I.J.; Airley, R.E. Prognostic value of facilitative glucose transporter GLUT 1 in oral squamous cell carcinomas treated by surgical resection; results of EORTC Translational Research Fund studies. Eur. J. Cancer 2004, 40, 503-507.

15. Hermanek, P., Sobin, L.H., Eds. UICC: TNM Classification of Malignant Tumors, 4th ed.; Springer: New York, NY, USA, 1987.

16. Masanori, Y.; Naoki, O.; Hiroyuki, H.; Yoichi, K.; Yohei, M.; Tetsuji, I.; Yusuke, M.; Keiichi, T.; Takeo, M.; Osamura, R. Glucose transporter-1 expression in the thyroid gland: clinocopathological significance for papillary carcinoma. Oncol. Rep. 2005, 14, 1499-504.

17. Tohma, T.; Okazumi, S.; Makino, H.; Cho, A.; Mochizuki, R.; Shuto, K.; Kudo, H.; Matsubara, K.; Gunji, H.; Matsubara, H.; Ochiai, T. Over expression of glucose transporter 1 in esophageal squamous cell carcinomas: a marker for poor prognosis. Dis. Esophagus 2005, 18, 185-189.

18. Zhou, S.; Wang, S.; Wu, Q.; Fan, J.; Wang, Q. Expression of glucose transporter-1 and -3 in the head and neck carcinoma-the correlation of the expression with the biological behaviors. ORL $J$. Otorhinolaryngol. Relat. Spec. 2008, 70, 189-194. 
19. Rocha, R.M.; Miller, K.; Soares, F.A.; Schenka, N.M.; Vassallo, J.; Gobbi, H. Biotin-free systems provide stronger immunohistochemical signal in oestrogen receptor evaluation of breast cancer. $J$. Clin. Pathol. 2009, 62, 699-704.

Sample Availability: No samples are available.

(C) 2010 by the authors; licensee Molecular Diversity Preservation International, Basel, Switzerland. This article is an open-access article distributed under the terms and conditions of the Creative Commons Attribution license (http://creativecommons.org/licenses/by/3.0/). 